\title{
THE ORIGIN OF COMMENSURABILITIES IN THE
}

\section{SATELLITE SYSTEMS}

\section{S. F. DER MOTT}

Department of Geophysics and Planetary Physics, The University, Newcastle upon Tyne, NEI 7RU, England

The distribution of orbits in the satellite systems of the major planets is definitely nonrandom as there is a marked preference for commensurability among pairs of mean motions in these systems. If this preference is not the result of a formation process then it follows that since the time of satellite formation dynamical evolution has occurred. In this paper (a full version of which is to be submitted to another journal) I show that there is a bulk of evidence in favour of the hypothesis that orbital evolution has occurred as the result of tidal dissipation in the planets. This evidence can be listed as follows:

(i) In a tidally evolved satellite system there should be a linear correlation between $\log$ (orbital radius) and $\log$ (satellite mass). The expected correlation is observed among the inner satellites of Saturn and Uranus, the slope of the log-log plots being consistent with an amplitude and frequency independent $Q$ (the tidal dissipation function). The correlation in Saturn's system must be largely due to either a formation process or possibly an early amplitude and frequency dependent tidal process, but certainly the present satellite distribution is completely consistent with the tidal hypothesis.

(ii) If $Q$ is amplitude- and frequency-independent then the Mimas-Tethys and Enceladus-Dione resonances are stable under the action of tidal forces. This is an important result as it indicates that the necessary tidal dissipation occurred in the solid parts of the planets. Whether or not these planets have any solid parts at present is a matter of dispute.

(iii) The tidal hypothesis can account for the formation (capture into libration) of the Mimas-Tethys and Enceladus-Dione resonances.

(iv) If it is allowed that the age of the Mimas-Tethys resonance is not small $\left(>10^{8} \mathrm{yr}\right)$ in comparison with that of the solar system and that orbital evolution since the time of satellite formation has been appreciable then it can be shown that tidal forces (with $Q$ amplitude- and frequency-independent) can account for the present large amplitude of libration of this resonance.

(v) If tidal evolution in the satellite systems of Jupiter and Saturn has been appreciable then from the present orbits of Io and Mimas it can be deduced that $Q$ (Jupiter) $\sim 1.1 \times 10^{5}$ and $Q$ (Saturn) $\sim 1.2 \times 10^{5}$. As the masses of the two satellites differ by a factor $\sim 2000$ and the amplitudes of the tides they raise on their respective planets differ by a factor $\sim 5000$ this agreement in the $Q$-values is somewhat remarkable. But as the stability of the Mimas-Tethys and Enceladus-Dione resonances demands that $Q$ is amplitude- and frequency-independent and as the chemical com- 
positions and structures of Jupiter and Saturn are probably similar then one should expect the $Q$ 's of these planets to be similar and thus the result supports the tidal hypothesis.

(vi) Finally, as the values of $Q$ are so very high it would be remarkable if tidal evolution had not taken place.

It would appear that tidal forces alone can account for the observed preference for commensurability among pairs of mean motions in the satellite systems of the major planets. This does not mean that evolution due to point-gravitational forces has not occurred but it does mean that there is no need to invoke such forces to account for the above preference. These results obviously have cosmogonical importance and they may also be of interest to cosmologists. If the gravitational constant is varying inversely with the age of the Universe, $T$, then as Mimas must always have been above synchronous height it can be shown that $T \gtrsim 1.1 \times 10^{10} \mathrm{yr}$.

\section{DISCUSSION}

R. Greenberg: Your conclusions seem to depend on a very simple functional dependence of $Q$ on amplitude and frequency. Can you show that a more general expression would yield the same results?

S. F. Dermott: My analysis, using $d n / d t=c n^{x} \mu^{y}$, has shown that appreciable orbital evolution of Mimas can be reconciled with the observed large amplitude of libration if at present $1 / n(d n / d t)$ for Mimas is approximately the same as that for Tethys. This is true if the forces acting are tidal forces.

Y. Kozai: Is the mean motion of Mimas decreasing due to the tidal force by your theory? I asked this question because by analyzing data for $300 \mathrm{yr}$ I found that the mean longitude of Mimas had secular acceleration.

S. F. Dermott: Yes, it is. However, the amount of change is so small and it cannot be detected from the observations. 